\title{
Putting a recombinant extracellular peptide of human Fzd7 into the context of triple negative breast cancer
}

\author{
Zhaoli Zhou ${ }^{1,2}$, Wei Xie ${ }^{1,2}$, Yihan Qian ${ }^{3}$, Jin Wang ${ }^{2}$ and Gang Huang ${ }^{1 *}$ \\ ${ }^{1}$ Shanghai Key Laboratory for Molecular Imaging, Collaborative Scientific Research Center. Shanghai University of Medicine \& Health Science, Shanghai, China \\ ${ }^{2}$ Department of Pharmacology, Faculty School of Pharmacy, Shanghai University of Medicine \& Health Science, Shanghai, China \\ ${ }^{3}$ Faculty School of Pharmacy, Fudan University, Shanghai, China
}

\begin{abstract}
Triple negative breast cancer (TNBC) is one of those intractable malignancies, featured by dysregulated activation of Wnt/ $\beta$-catenin signaling pathway. A recent study conducted by Shanghai University of Medicine \& Health Science shows that a novel recombinant human Frizzled-7 protein (rhFzd7) has potential efficacy against triple negative breast cancer by inhibiting Wnt/ $\beta$-catenin pathway, which give insightful meaning for the development of Fzd7-targeted drugs against TNBC. rhFzd7 may serve as a pharmacological probe to explore further the function of FZD7 in cancer biology and develop FZD7-based antibody.
\end{abstract}

Purpose: The purpose of the study is to use the extracellular domain of human Fzd7 to develop a soluble recombinant peptide (named as rhFzd7), to decoy Wnt and inhibit the pathway, and to inhibit TNBC.

\section{Background}

Frizzled receptors (FZDs) are a family of seven-transmembranespanning proteins belonging to the superfamily of $\mathrm{G}$ protein-coupled receptors [1]. They are comprised of ten members (FZD1-FZD10), which anchor in cell membrane and function as receptors of Winglesstype (WNT) signaling pathway. Wnt/ $\beta$-catenin signaling pathway through FZDs play key roles in both normal development and tumorigenesis, especially they have been demonstrated to be involved in a large variety of cancer-related processes, such as proliferation, migration, invasion, angiogenesis, stemness, and chemoresistance after cancer recurrence. FZD7 is the most studied member in the FZDs family. Those findings highlight the biological role of FZD7 in cancer and indicate FZD7 being an attractive pharmacological target.

Different approaches have been designed to block FZD7, including small molecule inhibitors, small peptides, antibodies, and soluble FZD7 [2]. A recent review, titled as "Frizzled Receptors as Potential Therapeutic Targets in Human Cancers", give a list of those FZD7 antibodies/inhibitors under development, including two small molecule compounds SRI37892 and FJ9, two small interfering peptides RHPDs and dFz7-21 [3], two antibodies scFvs and OMP-18R5, and one soluble FZD7. OMP-18R5 (Vantictumab) is the only one currently in phase I clinical evaluation. The cancer type for those inhibitors are Wilms' tumor, breast cancer, pancreatic cancer, and hepatocellular carcinoma, et al.

Triple negative breast cancer (TNBC) is a type of breast cancer whose expression of ER, PR, and HER2 is negative. Clinically it is characterized as more aggressive and less responsive to standard treatment with a poorer overall patient prognosis. Recent studies indicate that $\mathrm{Wnt} / \beta$-catenin signaling is particularly activated in TNBC. FZD7 is frequently upregulated in TNBC cell lines and tissues. Knockdown of FZD7 with siRNA, or using scFvs against FZD7, can suppress tumor transformation and inhibit tumor growth of TNBC. One condition of the clinical trial of Vantictumab is for locally recurrent or metastatic breast cancer (In combination with Paclitaxel, NCT01973309).

In sum, targeting Wnt signal pathway, especially targeting Fzd7, is a promising strategy against TNBC. Those inhibitors under development will be helpful for us to clarify the pathway, such as their role in cancer, and the redundancy of each components in this pathway. They are also promising agents against the intractable cancer TNBC.

\section{Results and conclusion}

rhFzd7 (around 27kd) was generated using E. coli system, with the codons optimized by the preference of E. coli. This novel rhFzd7 was demonstrated to have specificity and affinity to Wnt3a, antagonize Wnt $/ \beta$-catenin pathway, and exhibit anti-tumor and anti-angiogenesis activity against TNBC. When used in combination with standard chemotherapeutic agent Docetaxel, rhFzd7 sensitized TNBC to Docetaxel significantly. These results give insightful meaning for the development of Fzd7-targeted drugs against TNBC. The peptide described here represents a promising small ligand that specifically targets FZD7, and it serves as a pharmacological probe to explore further the function of FZD7 in cancer biology and develop FZD7based antibody.

*Correspondence to: Gang Huang, Shanghai Key Laboratory for Molecular Imaging, Collaborative Research Center. Shanghai University of Medicine \& Health Science, Shanghai, China, E-mail: huanggang@sumhs.edu.cn

Key words: human Fzd7, breast cancer, Fzd7-targeted drugs, FZD7-based antibody, triple negative breast cancer

Received: November 28, 2018; Accepted: December 10, 2018; Published: December 12, 2018 


\section{References}

1. Nusse R, Clevers H (2017) Wnt/ $\beta$-Catenin Signaling, Disease, and Emerging Therapeutic Modalities. Cell 169 :985-999. [Crossref]

2. Zeng CM, Chen Z, Fu L (2018) Frizzled Receptors as Potential Therapeutic Targets in Human Cancers. Int J Mol Sci 19. [Crossref]
3. Nile AH, de Sousa E Melo F, Mukund S, Piskol R, Hansen S, et al. (2018) A selective peptide inhibitor of Frizzled 7 receptors disrupts intestinal stem cells. Nat Chem Biol 14: 582-590. [Crossref]

Copyright: (C2018 Zhou Z. This is an open-access article distributed under the terms of the Creative Commons Attribution License, which permits unrestricted use, distribution, and reproduction in any medium, provided the original author and source are credited. 